\section{Superior Longitudinal Fasciculus}

Randall E. Merchant

Department of Anatomy \& Neurobiology, Virginia Commonwealth University, Richmond, VA, USA

\section{Definition}

The superior longitudinal fasciculus is a pair of long bi-directional association fiber bundles connecting the frontal, occipital, parietal, and temporal lobes. These fibers are known to be involved in the regulation of motor behavior, spatial attention and visual oculomotor functions, transfer of somatosensory information between parietal and motor cortices, articulation of language, and integration of auditory information. 